\title{
Transport properties of silicon implanted with bismuth
}

\author{
E. Abramof* and A. Ferreira da Silva ${ }^{\dagger}$ \\ Instituto Nacional de Pesquisas Espaciais-INPE, Laboratório Associado de Sensores e Materiais-LAS, Caixa Postal 515, \\ 12201-970 São José dos Campos, São Paulo, Brazil \\ Bo E. Sernelius \\ Department of Physics, Linköping University, S-581 83 Linköping, Sweden \\ J. P. de Souza and H. Boudinov \\ Instituto de Física UFRGS, 91501-970 Porto Alegre, Rio Grande do Sul, Brazil
}

(Received 7 May 1996; revised manuscript received 20 October 1996)

\begin{abstract}
The Hall effect and resistivity of $\mathrm{Si}: \mathrm{Bi}$ with donor concentration varying from $3.0 \times 10^{17}$ to $1.4 \times 10^{20}$ $\mathrm{cm}^{-3}$ were measured from room temperature down to $13 \mathrm{~K}$. The samples were prepared by $\mathrm{Bi}^{+}$implantation in Van der Pauw structures delineated in Si chips. The measured resistivities were compared with the ones calculated by a generalized Drude approach at similar temperatures and doping concentration, presenting fairly good agreement. The critical impurity concentration $N_{c}$ of the metal-nonmetal transition was measured to be around $2 \times 10^{19} \mathrm{~cm}^{-3}$. The critical concentration $N_{c}$ was calculated by comparing the ionization energy of the insulating phase with the total energy of the metallic phase. This value of $N_{c}$ agreed very well with the one obtained experimentally and the values estimated from other theoretical approaches. [S0163-1829(97)01612-3]
\end{abstract}

\section{INTRODUCTION}

The interest in the experimental and the theoretical investigation of the electronic properties of $n$-doped silicon has recently increased. ${ }^{1-20}$ The importance of disorder and correlation effects around the impurity critical concentration $N_{c}$ for the metal-nonmetal (MNM) transition is still the subject of many investigation..$^{1-10}$

Bismuth-doped silicon ( $\mathrm{Si}: \mathrm{Bi})$, which has received much less attention than other donors in $\mathrm{Si}$, has a larger ionization energy (smaller effective Bohr radius) and consequently higher critical concentration $N_{c}$ when compared to the other group- $V$ donors (P, As, and $\mathrm{Sb}$ ). ${ }^{11}$ These characteristics make the Si:Bi system very appropriate for the theoretical modeling of the transport properties from the insulating to the metallic phase, as well as for the investigation of the different mechanisms responsible for the MNM transition.

In this work, the primary emphasis will be on the resistivity and Hall effect measurements in $\mathrm{Si}: \mathrm{Bi}$, from room temperature down to $13 \mathrm{~K}$, with variation of the impurity concentration in the insulator to metallic range. The measured samples were prepared by $\mathrm{Bi}^{+}$implantation in Van der Pauw structures delineated in Si chips. The band conduction and impurity conduction activation energies were obtained from the slopes of the two branches of the resistivity curves.

The resistivities obtained experimentally are compared with the ones calculated from a recently proposed generalized Drude approach (GDA) at similar temperatures and doping regimes. For the resistivity calculation, we start from a frequency-dependent general expression for a polar semiconductor, reducing it to a nonpolar one in the static regime considering the effects of scattering by a random distribution of Coulomb impurities. The value of the critical concentration $N_{c}$ is obtained by comparing the ionization energy in the insulating phase $E_{I}$ with the total energy in the metallic phase $E_{T}$. The ionization energy $E_{I}$ is calculated using the dielectric function model with a Lorentz-Lorenz correction. This value of $N_{c}$ obtained in such a general mode is compared with the ones estimated from other theoretical approaches and the experimental value determined during this work.

\section{EXPERIMENT}

We use $p$-type, (100)-oriented Si wafers with resistivity in the range 16-25 $\Omega \mathrm{cm}$. Bismuth was implanted at room temperature in Van der Pauw structures ${ }^{21}$ with contact areas presenting low sheet resistance $(<20 \Omega / \square$, phosphorous doped). Five implantation with energies of 360, 200, 120, 70, and 35 $\mathrm{keV}$ were accumulated in each sample with proper doses to result in a plateaulike profile of $\mathrm{Bi}$ from the surface to the depth of $0.12 \mu \mathrm{m}$ with $\sim 5 \%$ deviation, according to TRIM code simulation. ${ }^{22}$

The implanted $\mathrm{Bi}$ doses were $2.5 \times 10^{15} \mathrm{~cm}^{-2}$ (at 360 $\mathrm{keV}$ ), $1.4 \times 10^{15} \mathrm{~cm}^{-2}$ (at $200 \mathrm{keV}$ ), $8 \times 10^{14} \mathrm{~cm}^{-2}$ (at 120 $\mathrm{keV}$ ), $7 \times 10^{14} \mathrm{~cm}^{-2}$ (at $70 \mathrm{keV}$ ), and $5 \times 10^{14} \mathrm{~cm}^{-2}$ (at 35 $\mathrm{keV}$ ) in order to achieve a Bi concentration of $5 \times 10^{20} \mathrm{~cm}^{-3}$. The doses in the other samples were scaled to this sample with $5 \times 10^{20} \mathrm{~cm}^{-3}$, according to the ratio of the desired $\mathrm{Bi}$ concentration. Samples with implanted Bi concentration from $5 \times 10^{17}$ to $5 \times 10^{20} \mathrm{~cm}^{-3}$ were prepared.

The damage annealing and the electrical activation of $\mathrm{Bi}$ were performed at $600{ }^{\circ} \mathrm{C}$ for $30 \mathrm{~min}$ in inert atmosphere in a halogen lamp furnace. For samples with Bi concentration less than $1 \times 10^{19} \mathrm{~cm}^{-3}$, a second annealing step was performed at $900{ }^{\circ} \mathrm{C}$ for $60 \mathrm{~s}$. The annealing process is described in detail elsewhere. ${ }^{2,23}$

The as-implanted and annealed samples were analyzed by Rutherford backscattering spectrometry using $760-\mathrm{keV} \mathrm{He}^{2+}$ beam in random and in (100)-aligned (RBS/C) incidence di- 


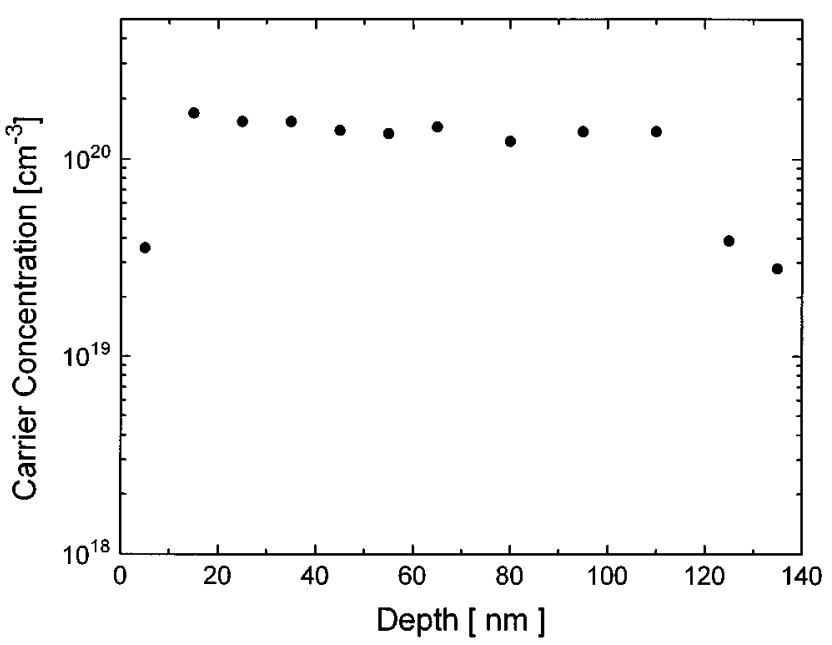

FIG. 1. Carrier depth profile of a sample implanted with the highest $\mathrm{Bi}$ dose $\left(5.9 \times 10^{15} \mathrm{~cm}^{-2}\right)$ after annealing at $600{ }^{\circ} \mathrm{C}$ for 30 min.

rections. The carrier concentration depth distribution was obtained in the annealed Van der Pauw devices via sequential anodic oxidation and oxide striping followed by sheet resistance and Hall effect measurements.

The electrical measurements were performed in an automated Keithley 180A Hall effect system. The Van der Pauw $\mathrm{Si} B \mathrm{Bi}$ samples were mounted in a He closed-cycle cryostat that allowed Hall effect and resistivity measurements from room temperature down to $13 \mathrm{~K}$. The measured electron concentration of the prepared samples at $290 \mathrm{~K}$ ranged from $3.0 \times 10^{17}$ to $1.4 \times 10^{20} \mathrm{~cm}^{-3}$; these values are considered in this work as the donor impurity concentration $N_{d}$ of the samples.

\section{RESULTS}

RBS/C analysis revealed that the as-implanted sample with the lower Bi dose (total dose of $5.9 \times 10^{12} \mathrm{~cm}^{-2}$ ) is crystalline with defect concentration level of $10 \%$ in a layer extending from the surface up to the depth of $0.12 \mu \mathrm{m}$. In other samples the Bi-implanted layer was rendered amorphous.

The layer implanted to the highest $\mathrm{Bi}$ dose (total dose of $5.9 \times 10^{15} \mathrm{~cm}^{-2}$ ) was epitaxially recrystallized during annealing, presenting a residual defect concentration below the detection level of our RBS/C analysis (less than $1 \%$ ). However, a highly defective layer (thickness of $20 \mathrm{~nm}$ ) remained at the sample surface. In the other samples the residual defect concentration after annealing is below the detection level along all the depth.

Figure 1 shows the carrier concentration depth profile of the sample implanted with the highest total $\mathrm{Bi}$ dose $\left(5.9 \times 10^{15} \mathrm{~cm}^{-2}\right)$ after annealing. The profile is flat topped, extending from a depth of $10 \mathrm{~nm}$ up to $120 \mathrm{~nm}$, with an average concentration of $1.4 \times 10^{20} \mathrm{~cm}^{-3}$. This value represents an electrical activation of about $28 \%$ of the implanted $\mathrm{Bi}$ atoms. It is interesting to point out that this activated concentration is three orders of magnitude higher than the solid solubility of $\mathrm{Bi}$ in $\mathrm{Si}$ at $1100{ }^{\circ} \mathrm{C}^{24}$ Such a highly activated $\mathrm{Bi}$ concentration has been previously observed to form

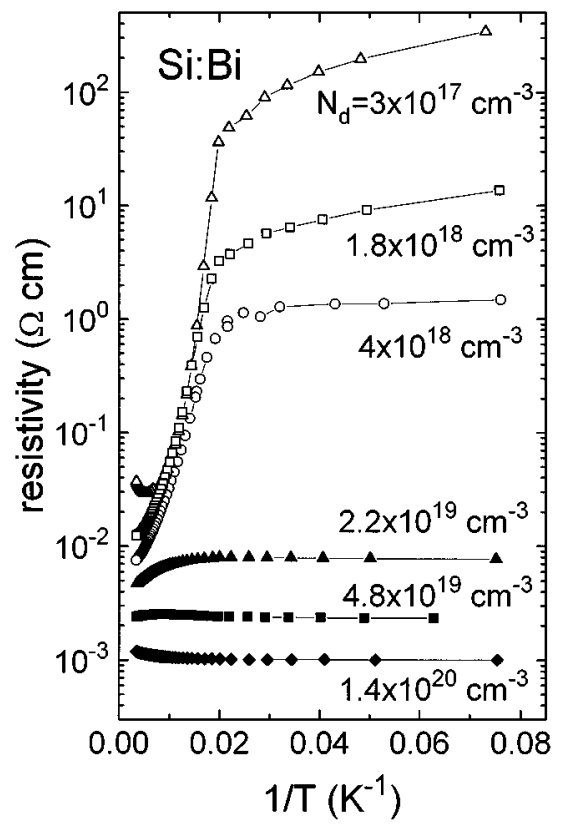

FIG. 2. Electrical resistivity of Si:Bi with different donor impurity concentration $N_{d}$ versus the inverse temperature.

during solid-phase epitaxial regrowth of an amorphized layer and presents metastability during annealing. ${ }^{23}$

Figures 2 and 3 show the electrical resistivity and Hall coefficient of the Si:Bi samples with different donor impurity concentration $N_{d}$ as a function of temperature. The metallic character of the samples with $N_{d}$ higher than $2 \times 10^{19} \mathrm{~cm}^{-3}$ (three curves in the bottom part of both graphs) is evidenced by the temperature-independent values of both resistivity and Hall coefficient through the whole temperature range investigated.

For samples with Bi donor concentration less than $2 \times 10^{19}$

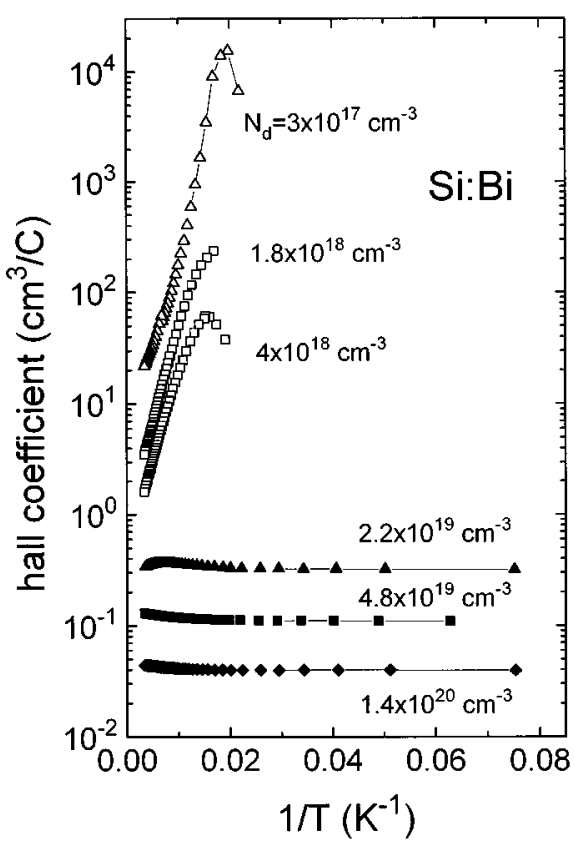

FIG. 3. Temperature dependence of the Hall coefficient of Si:Bi samples with different donor concentration $N_{d}$. 
TABLE I. Band conduction activation energy $E_{1}$ and the impurity conduction activation energy $E_{2}$ for $\mathrm{Si}$ :Bi.

\begin{tabular}{lcc}
\hline \hline$N_{d}\left(\mathrm{~cm}^{-3}\right)$ & $E_{1}\left(10^{-3} \mathrm{eV}\right)$ & $E_{2}\left(10^{-3} \mathrm{eV}\right)$ \\
\hline $3.0 \times 10^{17}$ & 70.6 & 2.2 \\
$1.8 \times 10^{18}$ & 40.2 & 1.5 \\
$4.0 \times 10^{18}$ & 31.6 & 0.3 \\
\hline
\end{tabular}

$\mathrm{cm}^{-3}$, the large enhancement in the resistivity and Hall coefficient as the temperature decreases, due to the reduction of free electrons in the conduction band, demonstrates the semiconductor properties of the $\mathrm{Si}: \mathrm{Bi}$ samples in this range of $N_{d}$. At the temperature where the impurity conduction starts to replace the band conduction transport, the Hall coefficient curves of these samples show a maximum and the resistivity curves change their slopes drastically. As shown in Fig. 3, the maximum in the Hall coefficient curves shifts to higher temperatures as $N_{d}$ increases.

The band conduction activation energy $E_{1}$ and the impurity conduction activation energy $E_{2}$ calculated from the slopes of the two branches of the $\log _{10} \rho$ vs $1 / T$ curves are shown in Table I for the three semiconducting samples. The value $E_{1}=71 \mathrm{meV}$, considered as the donor ionization energy of $\mathrm{Bi}$ in $\mathrm{Si}^{11}{ }^{11}$ was found only for the most diluted sample with $N_{d}=3 \times 10^{17} \mathrm{~cm}^{-3}$. As the donor concentration $N_{d}$ increases towards the critical concentration $N_{c}$, the measured activation energy $E_{1}$ decreases its value, as expected in such a MNM transition. ${ }^{25}$ The values of $E_{2}$ shown in Table I were calculated in the part of the curve where $1 / T$ is higher than $0.03 \mathrm{~K}^{-1}$, as only at these temperatures the slopes approach a constant value. These values are very small, showing an almost metallic impurity conduction. This fact was also observed for GaAs:Mn, which is an acceptor with almost the same ionization energy of $\mathrm{Bi}$ in $\mathrm{Si}^{26}$ The activation energy $E_{2}$ also decreases as $N_{d}$ increases, showing the same behavior as for $E_{1}$.

Figure 4(a) shows the resistivity dependence of $\mathrm{Si}: \mathrm{Bi}$ on the donor impurity concentration $N_{d}$ at different temperatures. We can observe that all the curves $\rho$ vs $N_{d}$ for different temperatures merge together to one critical point. This plot indicates clearly that the critical impurity concentration $N_{c}$ for the metal-nonmetal transition is around $2 \times 10^{19} \mathrm{~cm}^{-3}$ for silicon doped with bismuth. Figure 4(b) shows the theoretical resistivity of $\mathrm{Si}: \mathrm{Bi}$ calculated from the generalized Drude approach with the same donor concentration and temperatures of Fig. 4(a). The calculations using the GDA method to determine the resistivity and the theoretical determination of the critical concentration of $\mathrm{Si}: \mathrm{Bi}$ are described in the next section.

\section{THEORY AND DISCUSSION}

In order to calculate the dependence of the resistivity on temperature and impurity concentration, we use the generalized Drude approach. In the GDA, one starts from a generalized Drude expression for the dynamical conductivity ${ }^{20,27,28}$

$$
\sigma(\omega)=\frac{N e^{2}}{m^{*}} \frac{1}{1 / \tau(\omega)-i \omega},
$$

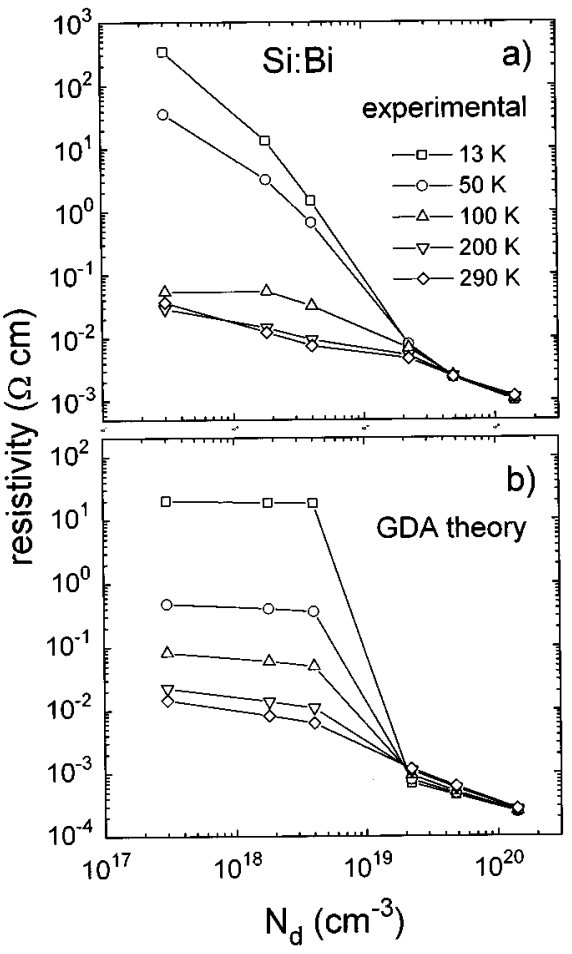

FIG. 4. Si:Bi resistivity as a function of the donor concentration $N_{d}$ (a) measured at different temperatures and (b) calculated by the generalized Drude approach at the same $N_{d}$ values and temperatures.

with a generalized complex-valued and frequency-dependent relaxation time $\tau(\omega)$. The expression found for $\tau(\omega)$ is valid for all frequencies, in particular for zero frequency, which is what we are interested in here. The net effect of the GDA is derived in the more general case of a polar semiconductor. ${ }^{27,29}$ The resistivity is then obtained as $1 / \sigma$ and is found to $\mathrm{be}^{27}$

$$
\begin{aligned}
\rho(\omega)= & \frac{-i m^{*} \omega}{N_{d} e^{2}}-\frac{i 2}{3 \pi N_{d} \omega} \\
& \times \int_{0}^{\infty} q^{2} \frac{[\alpha(q, \omega)-\alpha(q, 0)]\left[\varepsilon_{L}(q, \omega)+\alpha(q, 0)\right]}{\varepsilon_{T}^{2}(q, 0) \varepsilon_{T}(q, \omega)} d q .
\end{aligned}
$$

In the above expression, $N_{d}$ is the donor impurity concentration, $e$ is the electric charge, $m^{*}$ is the effective mass, $\alpha$ is the polarizability from the carriers, $\varepsilon_{L}$ is the lattice dielectric function, and $\varepsilon_{T}$ is the total dielectric function. ${ }^{27}$

For frequency independent $\varepsilon_{L}$ and no phonon polarizability, Eq. (2) reduces to the expression for a nonpolar semiconductor ${ }^{2,18}$

$$
\rho(\omega)=\frac{-i m^{*} \omega}{N_{d} e^{2}}+\frac{i 2}{3 \pi N_{d} \omega} \int_{0}^{\infty} q^{2}\left[\frac{1}{\varepsilon_{T}(q, \omega)}-\frac{1}{\varepsilon_{T}(q, 0)}\right] d q,
$$

which is identical to the result obtained in the so-called energy loss method. ${ }^{30}$ We have assumed a random distribution of Coulomb impurities.

The dielectric function $\varepsilon_{T}$ is given by 


$$
\varepsilon_{T}(q, \omega)=\varepsilon+v \alpha_{1}(q, \omega)+i v \alpha_{2}(q, \omega),
$$

where $\varepsilon$ is the dielectric constant for $\mathrm{Si}$ and $\alpha_{1}$ and $\alpha_{2}$ are the real and imaginary parts of the polarizabilities from one of the $v$ valleys of dopant carriers. The number of valleys is 6 in $\mathrm{Si}$, and these functions are temperature dependent.

We are interested in the static resistivity, which can be written as

$$
\rho(0)=\frac{16 \hbar k_{F}^{3} v}{12 \pi N_{d} E_{F}} \int_{0}^{\infty} Q^{2} \frac{\left.\frac{\partial \alpha_{2}(Q, W)}{\partial W}\right|_{W=0}}{\left[\varepsilon+v \alpha_{1}(Q, 0)\right]^{2}} d Q
$$

The imaginary part can be obtained analytically in the random-phase approximation. ${ }^{31}$ It is given by

$$
\alpha_{2}(Q, W)=-\frac{m^{*} e^{2}}{8 \hbar k_{F} Q^{3} B}\left[\ln \left(\frac{\cosh \left\{B\left[W+\left(Q^{2}+W^{2} / Q^{2}-M\right) / 2\right]\right\}}{\cosh \left\{B\left[W-\left(Q^{2}+W^{2} / Q^{2}-M\right) / 2\right]\right\}}\right)-2 B W\right],
$$

where we have introduced the dimensionless variables $Q=q / 2 k_{F}, W=\hbar \omega / 4 E_{F}, B=\beta E_{F}$, and $M=\mu / E_{F}$. The quantity $k_{F}$ is the Fermi wave number, $E_{F}$ is the Fermi energy, $\mu$ is the chemical potential, and $\beta=1 / K_{B} T$, where $K_{B}$ is the Boltzmann constant. The real part can be obtained from the imaginary part through the Kramers-Kronig dispersion relation.

The resistivity is reduced to

$$
\rho(0)=\frac{2 v\left(m^{*} e\right)^{2}}{3 \pi N_{d} \hbar^{3} k_{F}} \int_{0}^{\infty} \frac{\left\{1-\tanh \left[0.5 B\left(Q^{2}-M\right)\right]\right\}}{Q\left[\varepsilon+v \alpha_{1}(Q, 0)\right]} d Q
$$

The temperature variation of $\mu$ is obtained from the implicit expression $^{18,32}$

$$
B^{3 / 2}=\int_{0}^{U} \frac{3 y}{1-y^{2}}\left\{A+\ln \left[\left(1-y^{2}\right) / y^{2}\right]\right\}^{1 / 2} d y,
$$

where $U=\left(1+e^{-A}\right)^{-1 / 2}$ and $A=B M=\mu \beta$. For a given $A$ one obtains $B$. This means that a relation between $A$ and $B$ is found.

The calculated resistivity of $\mathrm{Si}: \mathrm{Bi}$ as a function of impurity concentration and temperature obtained using the procedure described above is presented in Fig. 4(b). For comparison, the resistivities of $\mathrm{Si}: \mathrm{Bi}$ were calculated at the same donor concentration and temperatures of the measured samples [Fig. 4(a)]. In both graphs, the resistivity at different temperatures converge to one common value at the same donor concentration around $2 \times 10^{19} \mathrm{~cm}^{-3}$, which is determined to be the critical concentration $N_{c}$ for the MNM transition in Si:Bi. Despite the differences in the absolute resistivity values, both measured and calculated $\rho$ vs $N_{d}$ curves presented similar forms. However, a qualitative difference between the theoretical and experimental curves is observed for the low-density region at low temperatures. The theoretical curves show a much weaker density dependence than the experimental ones. This is probably due to the neglect of multiple scattering in the theory. Multiple scattering, which leads to an enhancement of the resistivity, becomes of increasing importance at lower carrier concentration and temperature.

To determine the impurity critical concentration for the MNM transition we use a model with one localized donor level below the conduction band. The carriers are distributed throughout this level and the continuum states according to the Fermi-Dirac distribution function. We compare the energy in the insulating phase, the ionization energy $E_{I}$, with the energy per electron in the metallic phase $E_{T}$. The later one includes the kinetic energy, the exchange and correlation energies, and the electron-ion interaction energy. The reader should refer to Refs. 33 and 34 for more details about the derivation of this total energy.

The ionization energy $E_{I}$ is determined using the Lorentz expression for the dielectric function with the LorentzLorenz correction. The dielectric function for silicon plus unionized donors is

$$
\varepsilon(\omega)=1+\frac{\frac{4 \pi N_{u} \alpha_{d} \omega_{0}^{2}}{\omega_{0}^{2}-\omega^{2}-i \Gamma \omega}+4 \pi N_{h} \alpha_{h}}{1-\frac{1}{3}\left(\frac{4 \pi N_{u} \alpha_{d} \omega_{0}^{2}}{\omega_{0}^{2}-\omega^{2}-i \Gamma \omega}+4 \pi N_{h} \alpha_{h}\right)},
$$

where $N_{u}$ is the density of unionized donors, $N_{h}$ is the contribution of the host charge density, $E_{0}=\hbar \omega_{0}$ is the ionization energy for a single donor, and $\alpha_{d}$ and $\alpha_{h}$ are the contribution from the static polarizabilities for the unionized donors and host, respectively.

Developing the expression for $\varepsilon(\omega)$, the ionization frequency $\omega_{I}$ can be identified as

$$
\omega_{I}=\omega_{0}\left(1-\frac{4 \pi(\kappa+2) N_{u} \alpha_{d}}{9}\right)^{1 / 2},
$$

where

$$
\kappa=1+\frac{4 \pi N_{h} \alpha_{h}}{1-\frac{1}{3}\left(4 \pi N_{h} \alpha_{h}\right)} .
$$

The ionization energy $E_{I}=\hbar \omega_{I}$ as a function of $N_{u}$ is written as

$$
E_{I}=E_{0}\left[1-\frac{\pi(\kappa+2) N_{u}}{4}\left(\frac{e^{2}}{\kappa E_{0}}\right)^{3}\right]^{1 / 2},
$$

where we have used $\alpha_{d}=9 / 2 a_{d}^{3}$ and the Bohr radius for the donor $a_{d}=e^{2} / 2 \kappa E_{0}$, expressed as a function of the ionization energy for an isolated donor $E_{0}$. The density of unionized donors $N_{u}$ is related to the donor impurity concentration 


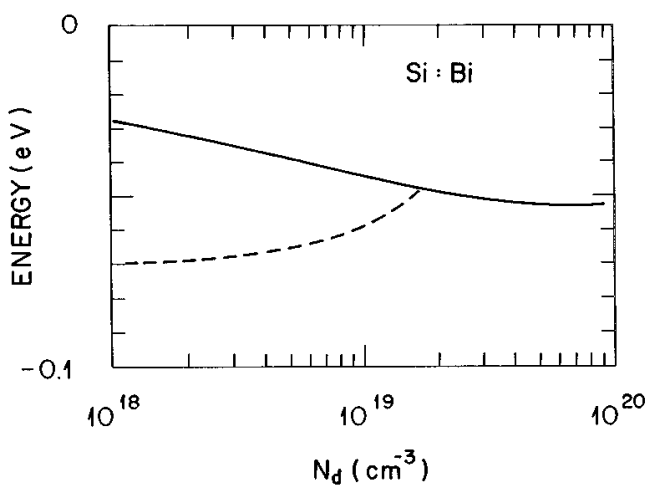

FIG. 5. Ionization energy of the insulating phase $E_{I}$ (dashed curve) and the energy of the metallic phase $E_{T}$ (solid curve) of $\mathrm{Si}$ :Bi versus the donor concentration $N_{d}$.

$N_{d}$ according to the Fermi-Dirac distribution with the chemical potential $\mu$ determined by Eq. (8).

The ionization energy in the insulating phase $E_{I}$ for $\mathrm{Si}: \mathrm{Bi}$ was calculated using Eq. (12) and assuming the experimental value of $71 \mathrm{meV}$ for $E_{0}$. This value of $71 \mathrm{meV}$ is considered to be the donor ionization energy of $\mathrm{Bi}$ in $\mathrm{Si}$ and was measured in this work for the most diluted sample (first $E_{1}$ value in Table I). Figure 5 shows the ionization energy of the insulating phase $E_{I}$ (dashed curve) and the energy of the metallic phase $E_{T}$ (solid curve) of $\mathrm{Si} \mathrm{Bi}$ as a function of the impurity donor concentration $N_{d}$. At the point where these energies cross, we obtain the critical concentration $N_{c}$ for the MNM transition. The value $N_{c}$ obtained in the crossing point is $1.69 \times 10^{19} \mathrm{~cm}^{-3}$.

The critical concentration $N_{c}$ can be obtained in a simpler way instead of the complex method of calculating and com-

*Electronic address: abramof@las.inpe.br

${ }^{\dagger}$ Also at Department of Physics, Linköping University, S581 83 Linköping, Sweden.

${ }^{1}$ S. Bogdanovich, Peihua Dai, M. P. Sarachik, and V. Dobrosavljevic, Phys. Rev. Lett. 74, 2543 (1995).

${ }^{2}$ A. Ferreira da Silva, Bo E. Sernelius, J. P. de Souza, and H. Boudinov, J. Appl. Phys. 79, 3453 (1996).

${ }^{3}$ T. G. Castner, Phys. Rev. B 52, 12434 (1995).

${ }^{4}$ Peihua Dai, Youzhu Zhang, and M. P. Sarachik, Phys. Rev. B 52, 12439 (1995).

${ }^{5}$ A. Gaymann, H. P. Geserich, and H. v. Löhneysen, Phys. Rev. B 52, 16486 (1995).

${ }^{6}$ M. Straub, K. Kirch, and H. v. Löhneysen, Z. Phys. B 95, 31 (1994).

${ }^{7}$ M. Hornung, A. Ruzzu, H. G. Schlager, H. Staupp, and H. v. Löhneysen, Europhys. Lett. 28, 43 (1994).

${ }^{8}$ T. G. Castner, Phys. Rev Lett. 73, 3600 (1994).

${ }^{9}$ Peihua Dai, Youzhu Zhang, and M. P. Sarachik, Phys. Rev. B 49, 14039 (1994).

${ }^{10}$ D. Belitz and T. Kirkpatrick, Rev. Mod. Phys. 66, 261 (1994).

${ }^{11}$ A. Ferreira da Silva, J. Appl. Phys. 76, 5249 (1994).

${ }^{12}$ A. Ferreira da Silva, Phys. Rev. B 50, 11216 (1994).

${ }^{13}$ A. Ferreira da Silva, Phys. Rev. B 48, 1921 (1993).

${ }^{14}$ G.-J. Yi and G. F. Neumark, Phys. Rev. B 48, 17043 (1993).

${ }^{15}$ M. J. Hirsch, D. F. Holcomb, R. N. Bhatt, and M. A. Paalanen, paring $E_{I}$ with $E_{T}$ by just finding the donor concentration where $E_{I}$ vanishes in Eq. (12), replacing $\kappa$ by the dielectric constant $\varepsilon$ and assuming $N_{u}=N_{d}$. We found $N_{c}=1.69 \times 10^{19}$ and $1.88 \times 10^{19} \mathrm{~cm}^{-3}$ for $\varepsilon=11.4$ and 12.0 , respectively, with the same $E_{0}=71 \mathrm{meV}$ of $\mathrm{Bi}$ in $\mathrm{Si}$. Both values of $N_{c}$ are very close to a value estimated previously, ${ }^{11}$ i.e., $N_{c}=1.8 \times 10^{19}$ $\mathrm{cm}^{-3}$. These values of the impurity critical concentration $N_{c}$ of Si:Bi determined theoretically by different methods agree very well with the value determined experimentally in this work.

\section{CONCLUSION}

In summary, the resistivity and Hall effect of silicon implanted with bismuth with impurity concentrations in the insulator to metallic phase were investigated experimentally as a function of temperature. The generalized Drude approach used to calculate the concentration and temperaturedependent resistivity worked well for the Si:Bi system, presenting fairly good agreement with the experimental results. The model, in which the ionization energy of the insulating phase is compared with the total energy in the metallic phase, applied to determine the impurity critical concentration for the MNM transition of Si:Bi presented good results when compared with the experimental value obtained here and the estimated values derived by other theoretical approaches.

\section{ACKNOWLEDGMENTS}

The authors acknowledge FAPESP (Project No. 94/1798-3) and the Brazilian Research Council CNPq for the financial support. We also thank Professors M. Sarachik and H. v. Löhneysen for very helpful correspondence.

${ }^{16}$ U. Thomanschefsky and D. F. Holcomb, Phys. Rev. B 45, 13356 (1992).

${ }^{17}$ Peihua Dai, Youzhu Zhang, and M. P. Sarachik, Phys. Rev. Lett. 66, 1914 (1991).

${ }^{18}$ Bo E. Sernelius and E. Söderström, J. Phys.: Condens. Matter 3, 1493 (1991).

${ }^{19}$ Bo E. Sernelius, Phys. Rev. B 43, 7136 (1991).

${ }^{20}$ Bo E. Sernelius, Phys. Rev. B 41, 3060 (1990).

${ }^{21}$ L. J. Van der Pauw, Philips Res. Rep. 13, 1 (1958).

${ }^{22}$ J. F. Ziegler, J. P. Biersak, and U. Littmark, The Stopping and Ranges of Ion in Solids (Pergamon, New York, 1985), Vol. I.

${ }^{23}$ J. P. de Souza and P. F. P. Fichtner, J. Appl. Phys. 74, 119 (1993).

${ }^{24}$ F. A. Trumbore, Bell Syst. Tech. J. 39, 205 (1960).

${ }^{25} \mathrm{H}$. Fritszche, in The Metal-Nonmetal Transition in Doped Semiconductors, edited by L. R. Friedman and D. P. Tunstall (Scottish Universities Summer School in Physics, Edinburgh, 1979), p. 193.

${ }^{26}$ D. A. Woodbury and J. S. Blakemore, Phys. Rev. B 8, 3803 (1973).

${ }^{27}$ Bo E. Sernelius, Phys. Rev. B 36, 1080 (1987).

${ }^{28}$ Bo E. Sernelius, Phys. Rev. B 40, 12438 (1989).

${ }^{29}$ Bo E. Sernelius and M. Morling, Thin Solid Films 177, 69 (1989). 
${ }^{30}$ E. Gerlach, J. Phys. C. 19, 4585 (1986).

${ }^{31}$ R. Sirko and D. L. Mills, Phys. Rev. B 18, 4373 (1978).

${ }^{32}$ Bo. E. Sernelius, in Shallow Impurities in Semiconductors, Proceedings of the Third International Conference in Shallow Impurities in Semiconductors, edited by B. Monemar, IOP Conf. Proc. No. 95 (Institute of Physics and Physical Society, London,
1988), p. 137

${ }^{33}$ K.-F. Berggren and Bo E. Sernelius, Phys. Rev. B 24, 1971 (1981).

${ }^{34}$ Bo E. Sernelius and K.-F. Berggren, Philos. Mag. B 43, 115 (1981). 